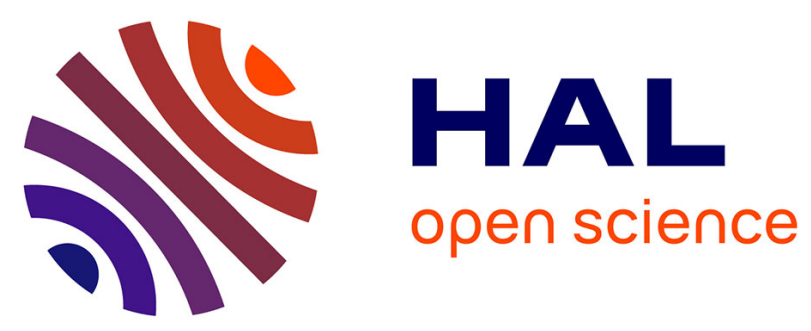

\title{
Optimisation de la permittivité complexe d'un liquide à partir de mesures interférométriques en ondes millimétriques
}

\author{
J. Goulon, G. Roussy, J.L. Rivail
}

\section{> To cite this version:}

J. Goulon, G. Roussy, J.L. Rivail. Optimisation de la permittivité complexe d'un liquide à partir de mesures interférométriques en ondes millimétriques. Revue de Physique Appliquée, 1969, 4 (3), pp.413-419. 10.1051/rphysap:0196900403041300 . jpa-00243305

HAL Id: jpa-00243305 https://hal.science/jpa-00243305

Submitted on 1 Jan 1969

HAL is a multi-disciplinary open access archive for the deposit and dissemination of scientific research documents, whether they are published or not. The documents may come from teaching and research institutions in France or abroad, or from public or private research centers.
L'archive ouverte pluridisciplinaire HAL, est destinée au dépôt et à la diffusion de documents scientifiques de niveau recherche, publiés ou non, émanant des établissements d'enseignement et de recherche français ou étrangers, des laboratoires publics ou privés. 


\title{
OPTIMISATION DE LA PERMITTIVITÉ GOMPLEXE D’UN LIQUIDE A PARTIR DE MESURES INTERFÉROMÉTRIQUES EN ONDES MILLIMÉTRIQUES
}

\author{
Par J. GOULON, G. ROUSSY et J. L. RIVAIL, \\ Équipe de Recherche associée au C.N.R.S., $\mathrm{n}^{0} 22$ (Interactions Moléculaires) \\ Laboratoire de Chimie Théorique, Faculté des Sciences de Nancy, I, rue Grandville, 54-Nancy.
}

(Reçu le 10 février 1969, révisé le 27 mai 1969.)

\begin{abstract}
Résumé. - Les auteurs développent l'étude d'un interféromètre de Michelson en G.S.D. dans 1'approximation d'une propagation quasi optique non perturbée par des modes supérieurs. Le rôle d'inévitables réflexions ou T.O.S. parasites est précisé et l'équation des interférogrammes est "mise sous une forme analytique simple permettant d'optimiser la détermination de la permittivité complexe $\varepsilon^{*}$ pour les liquides, en ondes millimétriques. La méthode numérique proposée offre la possibilité d'une estimation directe des barres d'erreur affectant $\varepsilon^{\prime}$ et $\varepsilon^{\prime \prime}$, et pour les liquides étudiés, on donne :

$$
\frac{\Delta \varepsilon^{\prime}}{\varepsilon^{\prime}}<1 \%, \frac{\Delta \varepsilon^{\prime \prime}}{\varepsilon^{\prime \prime}}<2 \% \text { à } \lambda=4,35 \mathrm{~mm} .
$$

Abstract. - A Michelson interferometer in oversized waveguide is studied, in the assumption of no propagating spurious modes. The influence of disturbing reflections or W.S.V.R. has been analysed and equations for the interferogramms are obtained which allow the authors to propose a numerical method of optimizing the determination of the complex permittivity of liquids in $\mathrm{mm}$. waves. An attempt is also made to evaluate directly the accuracy of the results which is higher than $1 \%$ for $\varepsilon^{\prime}, 2 \%$ for $\varepsilon^{\prime \prime}$, in the case of some liquids at $\lambda=4.35 \mathrm{~mm}$.
\end{abstract}

I. Introduction. - Dans un article récent [1], nous avons décrit la réalisation d'un interféromètre de Michelson en guide surdimensionné (G.S.D.), et nous avons déterminé la permittivité complexe $\varepsilon^{*}$ de divers liquides, avec une précision estimée à $2 \%$ pour $\varepsilon^{\prime}$, $4 \%$ pour $\varepsilon^{\prime \prime}$, à la longueur d'onde $\lambda=4,35 \mathrm{~mm}$, en exploitant nos interférogrammes expérimentaux par la méthode graphique proposée par Leroy et al. [2].

Nous pensons qu'il est possible de réduire ces barres d'erreur en tirant un meilleur parti du grand nombre d'informations fournies par le relevé point par point (de 100 à 400 points) des divers interférogrammes. Le but du présent article est de proposer une méthode numérique originale d'optimisation de $\varepsilon^{*}$ qui permette de réduire considérablement la part des erreurs de nature purement aléatoire, et d'affiner les déterminations parfois hasardeuses (en particulier dans le cas de liquides relativement absorbants) obtenues par la méthode de Leroy et al.

En premier lieu, nous chercherons à donner aux interférogrammes une forme mathématique plus élaborée que celle de Leroy et al., en tenant compte en particulier de l'existence d'inévitables réflexions parasites, mais en nous limitant, pour l'instant, toujours à l'approximation d'une propagation «quasi optique » non perturbée par des modes supérieurs.

La méthode d'optimisation proprement dite sera exposée ensuite et ses résultats seront discutés. Un mérite essentiel de cette nouvelle méthode est en effet de permettre une détermination semi-expérimentale des intervalles de confiance des valeurs de $\varepsilon^{\prime}$ et $\varepsilon^{\prime \prime}$ calculées.

II. Equation des interférogrammes. Rôle des réflexions parasites. - Le montage que nous avons

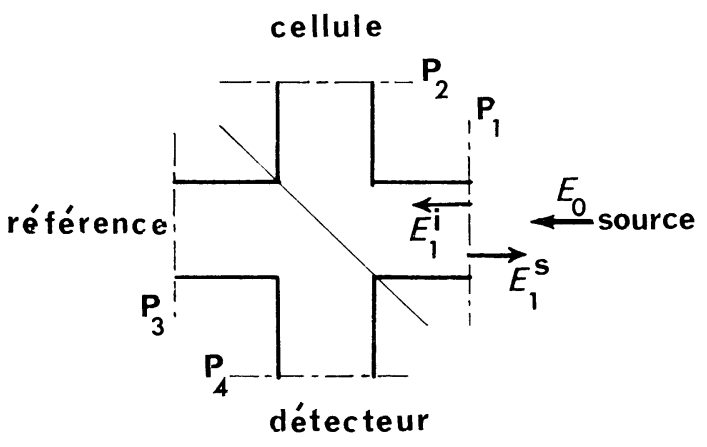

Fig. 1. - Schéma de l'octopole

équivalent à l'interféromètre de Michelson.

décrit utilise un coupleur en croix à lame semi-réfléchissante, que nous considérons comme un octopole (fig. 1) ${ }^{(1)}$.

(1) Les notations adoptées dans cet article sont celles de la référence [1]. 
Supposons que les amplitudes des ondes émergentes $E_{j}^{s}$ et des ondes incidentes $E_{j}^{i}$ soient mesurées dans le plan $P_{j}$ de la branche $j$. Nous avons la relation matricielle :

$$
\left\|E_{j}^{s}\right\|=\|S\| \cdot\left\|E_{j}^{i}\right\|
$$

|| $S \|$ étant la matrice de répartition de l'octopole (Scattering matrix, [3]).

Si nous admettons la propagation d'un seul mode, à une fréquence donnée, nous pouvons définir quatre plans $P_{j}$ de référence tels que la matrice de répartition de l'octopole sans perte s'écrive [4], [5] :

$$
\|S\|=\left\|\begin{array}{llll}
0 & r & j t & 0 \\
r & 0 & 0 & j t \\
j t & 0 & 0 & r \\
0 & j t & r & 0
\end{array}\right\| \text { avec } r^{2}+t^{2}=1
$$

$r, t$ désignant les coefficients de réflexion et de transmission en amplitude de la lame semi-réfléchissante sous une incidence de $45^{\circ}$.

L'absence de modes supérieurs nous autorise également à poser :

$$
\left\|\begin{array}{l}
E_{1}^{i} \\
E_{2}^{i} \\
E_{3}^{i} \\
E_{4}^{i}
\end{array}\right\|=\left\|\begin{array}{l}
\rho_{1} E_{1}^{s}+E_{0} \\
\rho_{2} E_{2}^{s} \\
\rho_{3} E_{3}^{s} \\
\rho_{4} E_{4}^{s}
\end{array}\right\|
$$

$\rho_{j}$ étant le coefficient de réflexion complexe, mesuré dans le plan $P_{j}$ du circuit $j$ extérieur au coupleur; $E_{0}$ étant l'amplitude de l'onde émise par la source et ramenée au plan $P_{1}$.

La relation matricielle (1) conduit au résultat : $E_{4}^{s} \cdot\left[1-r^{2}\left(\rho_{1} \rho_{2}+\rho_{3} \rho_{4}\right)+t^{2}\left(\rho_{1} \rho_{3}+\rho_{2} \rho_{4}\right)\right.$ $\left.+\left(r^{4}+t^{4}+2 r^{2} t^{2}\right) \rho_{1} \rho_{2} \rho_{3} \rho_{4}\right]=j r t E_{0}\left[\rho_{2}+\rho_{3}\right]$.

Les coefficients de réflexion $\rho_{1}$ et $\rho_{4}$ traduisent l'existence de réflexions parasites, et dans les conditions expérimentales on peut admettre que $\left|\rho_{1}\right|$ et $\left|\rho_{4}\right|$ sont des infiniment petits de même ordre :

$$
\left|\rho_{1}\right| \sim\left|\rho_{4}\right|=\delta \text {. }
$$

On obtient alors aisément le développement limité :

$$
S=E_{4}^{s} \cdot \bar{E}_{4}^{s}=\left(r t E_{0}\right)^{2} \cdot\left[\rho_{2}+\rho_{3}\right] \cdot\left[\bar{\rho}_{2}+\bar{\rho}_{3}\right]
$$$$
\left\{1+\delta\left(a_{1}+b_{1} \rho+\overline{b_{1} \rho}\right)+\delta^{2}\left(a_{2}+b_{2} \rho+\overline{b_{2} \rho}+c_{2}|\rho|^{2}\right)\right.
$$

$$
\left.+\delta^{3} \ldots\right\}
$$

où $\rho=\rho_{2}$ ou $\rho_{3}$ selon que l'on considère les interférogrammes $S(h)$ ou $S(d)$ [1].

Bien entendu, il suffit de faire $\delta=0$ dans cette expression (3) pour retrouver l'équation classique des interférogrammes en l'absence de réflexions parasites.

A. ÉQUATION DES INTERFÉROGRAMMES A VIDE $S(d)$. Les interférogrammes $S(d)$ sont obtenus, pour une position donnée du piston $M_{2}$, en déplaçant le piston de référence $M_{3}$ dans un guide sans pertes :

$\rho_{2}=$ Cte et $\rho=\rho_{3}=-\mathrm{e}^{-2 j \beta_{0} d}$ avec $\beta_{0}=\frac{2 \pi}{\lambda}$.
En reportant $\rho_{3}$ dans l'expression (3), nous obtenons :

$$
\begin{aligned}
S(d)=[A+ & \left.B \cos \left(2 \beta_{0} d+\psi\right)\right] \cdot\{1 \\
& +\delta\left[a_{1}+\left|b_{1}\right| \cdot \cos \left(2 \beta_{0} d+\psi_{1}\right)\right] \\
& +\delta^{2}\left[a_{2}+\left|b_{2}\right| \cdot \cos \left(2 \beta_{0} d+\psi_{2}\right)\right. \\
& \left.\left.+\left|d_{2}\right| \cos \left(4 \beta_{0} d+\psi_{2}^{\prime}\right)\right]+\delta^{3} \ldots\right\}
\end{aligned}
$$

Il apparaît donc clairement qu'en présence de réflexions parasites $(\delta \neq 0)$, ces interférogrammes $S(d)$ ne peuvent plus être sinusoïdaux, mais doivent, par contre, rester rigoureusement périodiques en $d$, et l'on pourrait envisager une décomposition en harmoniques spatiales. Ainsi se trouvent expliquées les anomalies signalées quant au profil de ces interférogrammes [1], [6].

A plus basse fréquence, les interférogrammes $S(d)$ cessent d'être périodiques. Ceci met en cause essentiellement l'hypothèse de la propagation d'un mode unique, comme nous avons pu le vérifier en réalisant des filtres de mode qui seront décrits ultérieurement.

B. ÉQuation des interfÉrogrammes $S(h)$. - Ces interférogrammes sont obtenus en déplaçant le piston $\mathrm{M}_{2}$ au sein du liquide, le piston $\mathrm{M}_{3}$ restant immobile. Ainsi :

$$
\rho_{3}=\text { Cte } ; \quad \rho=\rho_{2} \text {. }
$$

L'expression de $\rho=\rho_{2}$ est ici sensiblement plus compliquée, même lorsque la cellule est vide, en raison de la double discontinuité de milieu, créée par une fenêtre de téflon d'épaisseur non négligeable. Nous montrons dans l'annexe I que l'expression générale de $\rho_{2}$, pour une abscisse $h$ du piston $\mathbf{M}_{2}$, est de la forme :

$$
\rho_{2}(h)=\frac{P-Q X}{1-R X} \quad \text { avec } \quad X=\mathrm{e}^{-2 \alpha h} \cdot \mathrm{e}^{-2 j \beta h}
$$

où $P, Q, R$ sont trois coefficients complexes indépendants de $h$,

$\alpha+j \beta=\gamma_{2}$ est la constante de propagation du milieu 2 (cf. Annexe I).

Il est intéressant de développer $\rho_{2}(h)$ en série par rapport à $X$ et de reporter ce développement dans l'expression (3). On obtient ainsi l'équation théorique exacte des interférogrammes $S(h)$ sous la forme d'un développement en série dont les premiers termes sont :

$$
\begin{aligned}
S(h)=A & +\mathrm{e}^{-2 \alpha h}\{2 B \cdot \cos (2 \beta h)+2 C \cdot \sin (2 \beta h)\} \\
& +\mathrm{e}^{-4 \alpha h}\{D+2 E \cdot \cos (4 \beta h)+2 F \cdot \sin (4 \beta h)\} \\
& +\mathrm{e}^{-6 \alpha h}\{2 G \cdot \cos (2 \beta h)+2 H \cdot \sin (2 \beta h) \\
& +2 I \cdot \cos (6 \beta h)+2 J \cdot \sin (6 \beta h)\} \\
& +\mathrm{e}^{-8 \alpha h}\{\ldots\}
\end{aligned}
$$

$A, B, C \ldots$ sont des coefficients indépendants de $h$, que l'on peut difficilement expliciter en fonction de $\rho_{3}, P$, $Q, R, \delta$, mais qui sont des caractéristiques de l'appareil.

Ce développement a l'avantage d'expliciter sous forme d'harmoniques spatiales, simultanément :

- l'influence des discontinuités de milieu (fenêtre de téflon),

- l'existence de réflexions parasites, quelle que soit la substance contenue dans la cellule. 
Pour des corps suffisamment absorbants, et des épaisseurs importantes de liquides, $\mathrm{e}^{-2 n \alpha h}$ devient petit, et l'on peut se limiter aux premiers termes du développement, soit :

$$
\begin{gathered}
S(h)=A+\mathrm{e}^{-2 \alpha h}\{2 B \cdot \cos (2 \beta h)+2 C \cdot \sin (2 \beta h)\} \\
+\mathrm{e}^{-4 \alpha h}\{D+2 E \cdot \cos (4 \beta h)+2 F \cdot \sin (4 \beta h)\}
\end{gathered}
$$

ceci n'étant valable qu'au-delà d'une épaisseur $h_{0}$ de liquide que nous nous efforcerons de préciser par la suite.

III. Principe de l'analyse numérique des interférogrammes $S(h)$. - En admettant que les interférogrammes $S(h)$ sont de la forme $(6)$, il nous reste à déterminer les huit paramètres $\alpha, \beta, A, B, C, D, E, F$. Nous avons renoncé à calculer globalement ces huit paramètres par une méthode classique de moindres carrés, par exemple la méthode de Rosenbrock [7]. Le nombre élevé de paramètres à optimiser n'est pas le seul obstacle à une convergence satisfaisante de la méthode : la décroissance rapide des termes en $\exp (-4 \alpha h)$ est aussi une source de difficultés. Par ailleurs, l'initialisation des paramètres $B, C, D, E, F$ à partir des interférogrammes $S(h)$ est difficile.

Au contraire, il est assez facile d'obtenir graphiquement une première estimation des paramètres $\alpha, \beta, A$, en négligeant les termes en $\exp (-4 \alpha h)$ :

- $\alpha$ se déduit d'une représentation graphique de :

$$
\log \left|S_{\max }-S_{\min }\right|=2 \alpha h+\text { Cte. }
$$

- $\beta$ est donné par la pseudo-périodicité de la courbe $S(h)$.

- $A$ est la valeur limite de $S(h)$ pour $h$ grand; c'est aussi la valeur autour de laquelle $S(h)$ « oscille ».

C'est pourquoi il nous a semblé intéressant d'optimiser par étapes les huit paramètres.

A. Dans un premier temps, partant d'une première évaluation $\alpha^{0} \beta^{0} A^{0}$ de $\alpha, \beta, A$, on calcule un premier jeu de paramètres $B^{0} C^{0} D^{0} E^{0} F^{0}$ par la méthode d'estimation numérique dite « des moments ».

Les moments d'ordre $0,1,2$, de la fonction $S(h)$ sont :

$$
\begin{aligned}
Y_{\mathbf{1}} & =\int_{h_{0}}^{h_{1}}\left[S(x)-A^{0}\right] \mathrm{d} x \\
Y_{\mathbf{2}} & =\int_{h_{0}}^{h_{1}}\left[S(x)-A^{0}\right] \cos \left(2 \beta^{0} x\right) \mathrm{d} x \\
Y_{3} & =\int_{h_{0}}^{h_{1}}\left[S(x)-A^{0}\right] \sin \left(2 \beta^{0} x\right) \mathrm{d} x \\
Y_{4} & =\int_{h_{0}}^{h_{1}}\left[S(x)-A^{0}\right] \cos \left(4 \beta^{0} x\right) \mathrm{d} x \\
Y_{5} & =\int_{h_{0}}^{h_{1}}\left[S(x)-A^{0}\right] \sin \left(4 \beta^{0} x\right) \mathrm{d} x .
\end{aligned}
$$

Ces quantités peuvent s'exprimer linéairement en fonction des coefficients $B, C, D, E, F$ :

$$
\left\|Y_{j}\right\|=\|Z\| \cdot\left\|\begin{array}{l}
B \\
C \\
D \\
E \\
F
\end{array}\right\| \text {. }
$$

|| $Z$ || étant une matrice 5-5 dont les éléments sont calculés numériquement sur ordinateur et sont fonction de $\alpha^{0}, \beta^{0}, A^{0}, h_{0}, h_{1}$. La résolution du système (7) donne $B^{0}, C^{0}, D^{0}, E^{0}, F^{0}$ en fonction de ces mêmes paramètres.

B. Dans un deuxième temps, en affectant à $B, C$, $D, E, F$ les valeurs précédentes, on recherche une meilleure estimation de $\alpha, \beta, A$ que l'on peut recycler jusqu'à convergence satisfaisante de ces paramètres. On utilise pour cette deuxième étape du calcul la méthode du « maximum de vraisemblance ». Ainsi pour $N$ points de l'interférogramme, soit $N$ mesures indépendantes, on sait que le maximum de la fonction de vraisemblance s'obtient par la condition (8) :

$\Phi=\sum_{r=1}^{N}\left[S_{\text {expérimental }}^{r}-S_{\text {calculé }}^{r}\right]^{2}=$ minimum

$\Phi$ est une fonction des trois paramètres $\alpha, \beta, A$.

Fixons deux des variables, par exemple $\alpha$ et $\beta$, et calculons les valeurs de la fonction $\Phi$ pour les trois points $M_{1}(\alpha, \beta, A), M_{2}(\alpha, \beta, A-\Delta A), M_{3}(\alpha, \beta, A+\Delta A)$. Nous assimilons la section droite (obtenue pour $\alpha$ et $\beta$ constants) de la surface d'équation $\Phi$, à une parabole définie par les trois points $M_{1}, M_{2}, M_{3}$, et de sommet $M^{s}\left(\alpha, \beta, A^{s}\right)$ avec :

$$
A^{s}=A+\Delta A \cdot \frac{\Phi(\alpha, \beta, A-\Delta A)-\Phi(\alpha, \beta, A+\Delta A)}{2\{\Phi(\alpha, \beta, A-\Delta A)+\Phi(\alpha, \beta, A+\Delta A)-2 \Phi(\alpha, \beta, A)\}} .
$$

Le minimum de la section droite ainsi définie est donné par la valeur de la fonction $\Phi$ en $M^{s}$.

En effectuant le même calcul pour $\alpha$ et $\beta$, on définit ainsi trois points : $\left(\alpha^{s}, \beta, A\right),\left(\alpha, \beta^{s}, A\right),\left(\alpha, \beta, A^{s}\right)$. On obtient une meilleure estimation des paramètres $\alpha, \beta, A$, en choisissant parmi ces trois points celui qui correspond à la plus faible valeur de $\Phi$.

On peut reprendre alors l'ensemble du calcul jus- qu'à une convergence satisfaisante, visualisée par la décroissance de $\Phi$. L'expérience montre qu'elle est obtenue rapidement.

Le calcul de la permittivité $\varepsilon^{*}$ ne présente alors plus aucune difficulté puisque :

$$
\varepsilon^{\prime}=\frac{\beta^{2}-\alpha^{2}}{\beta_{0}^{2}} ; \quad \varepsilon^{\prime \prime}=\frac{2 \alpha \beta}{\beta_{0}^{2}} .
$$


IV. Mise en œuvre de la méthode de calcul. - Nous avons rédigé en Fortran IV un programme permettant l'exploitation de nos résultats sur ordinateur IBM-1130. La suite logique des opérations est indiquée dans un organigramme très simple.

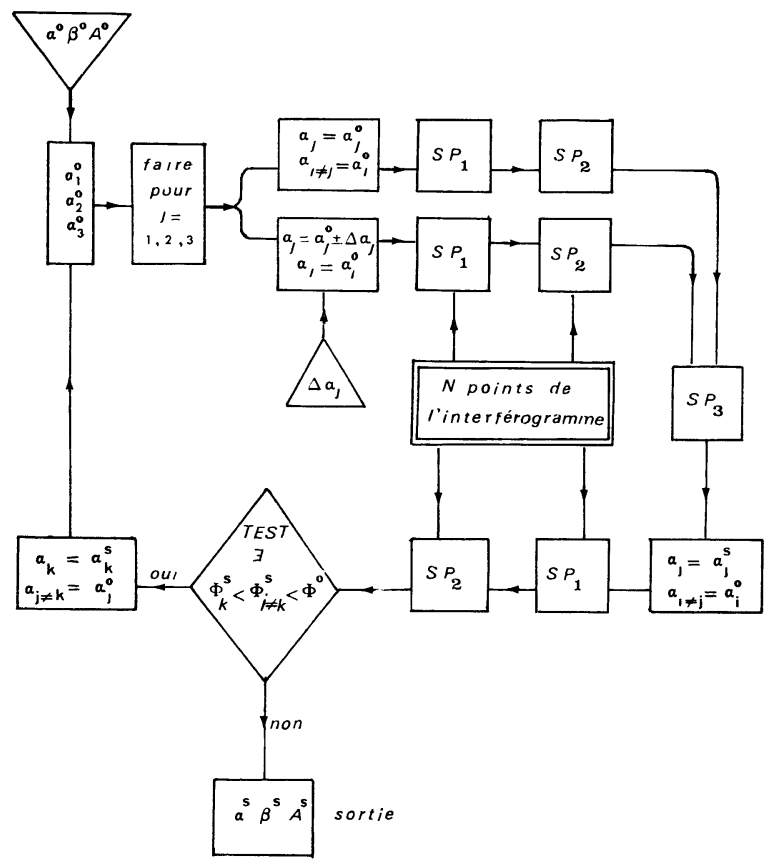

ORGANIGRAMME DE LA MÉTHODE DE CALCUL :

$\mathrm{SP}_{1}$ : Sous-programme de calcul des paramètres $B, C$, $D, E, F$ par la méthode des moments.

$\mathrm{SP}_{2}$ : Sous-programme de calcul de la fonction des moindres carrés $\Phi$.

$\mathrm{SP}_{3}$ : Sous-programme de calcul du sommet $\alpha_{j}^{s}$ de la parabole $\Phi\left(\alpha_{j}\right)$.
Pour essayer notre programme, nous avons simulé le cas d'un liquide de permittivité complexe très proche de celle du chloroforme, à $\theta=20^{\circ} \mathrm{C}$ et $\lambda=4,358 \mathrm{~mm}$ $\left(\varepsilon_{\text {exacte }}^{\prime}=2,6057, \varepsilon_{\text {exacte }}^{\prime \prime}=0,8539\right)$ et nous avons calculé à la précision maximum de l'ordinateur un « interférogramme théorique » de la forme (5). Partant alors de $N$ points de cet interférogramme calculé, et d'une estimation grossière de $\varepsilon^{\prime}, \varepsilon^{\prime \prime}, A$, le calcul converge rapidement vers des valeurs de $\varepsilon^{\prime}$ et $\varepsilon^{\prime \prime}$ dont l'accord avec les valeurs exactes dépend simultanément :

- du choix des bornes $h_{0}, h_{1}$ des intégrales intervenant dans le calcul des moments,

- des rectangles d'erreurs simulés pour les $N$ points.

La position du piston $h$ étant déterminée avec une très faible incertitude, nous admettrons qu'il est possible de remplacer les rectangles d'erreurs réels et simulés par de simples barres d'erreurs affectant les valeurs de $S$.

Pour chiffrer l'influence des barres d'erreurs sur $S$, nous avons effectué plusieurs fois le calcul, en admettant successivement pour $S$ un pouvoir de résolution $\left({ }^{2}\right)$ du système de détection de 10000,1000 et $100, h$ étant supposé connu exactement. Le tableau I regroupe les résultats de ces calculs pour des valeurs croissantes de $h_{0}, h_{1}$ demeurant constant.

Il apparaît clairement, pour chaque valeur du pouvoir de résolution, un seuil $h_{0 \max }$ au-delà duquel la précision du calcul de $\varepsilon^{\prime}, \varepsilon^{\prime \prime}$ chute rapidement. Cela s'explique simplement par l'amortissement des interférogrammes. Augmenter $h_{0}$ revient en effet à amputer l'interférogramme d'un domaine où l'amplitude des

(2) Voir norme AFNOR NF-X-07001, 1955.

TABLEAU I

\begin{tabular}{|c|c|c|c|c|c|c|c|}
\hline \multirow{2}{*}{\multicolumn{2}{|c|}{$\begin{array}{c}\text { PouvoIR } \\
\text { DE RÉSOLUTION } \\
\text { DU SYSTÈME } \\
\text { DE DÉTEGTION }\end{array}$}} & \multicolumn{6}{|c|}{$h_{0}(\mathrm{~mm})$} \\
\hline & & 0,05 & 0,55 & 1,05 & 1,35 & 2,05 & 2,55 \\
\hline - & & - & - & - & - & - & - \\
\hline \multirow{2}{*}{10000} & $\varepsilon^{\prime}$ & 2,605 & 2,605 & 2,605 & 2,604 & 2,604 & 2,603 \\
\hline & $\varepsilon^{\prime \prime}$ & 0,854 & 0,858 & 0,859 & 0,858 & 0,859 & 0,860 \\
\hline \multirow{2}{*}{1000} & $\varepsilon^{\prime}$ & 2,604 & 2,603 & 2,602 & 2,600 & 2,598 & 2,600 \\
\hline & $\varepsilon^{\prime \prime}$ & 0,855 & 0,857 & 0,858 & 0,859 & 0,858 & 0,857 \\
\hline \multirow{2}{*}{100} & $\varepsilon^{\prime}$ & 2,594 & 2,595 & 2,592 & 2,573 & 2,598 & 2,610 \\
\hline & $\varepsilon^{\prime \prime}$ & 0,869 & 0,883 & 0,891 & 0,928 & 0,938 & 0,947 \\
\hline
\end{tabular}




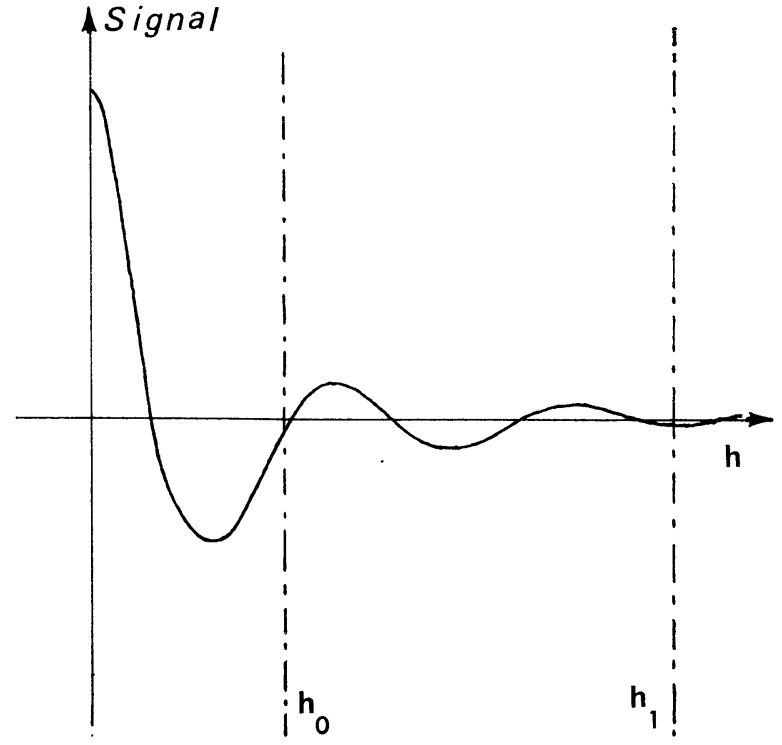

FIG. 2. - Influence de l'amortissement des interférogrammes sur le choix de $h_{0}$.

oscillations est maximum ( fig. 2). On conçoit ainsi que la précision du calcul de $\varepsilon^{\prime}, \varepsilon^{\prime \prime}$ soit bien plus influencée par le choix de $h_{0}$ que par celui de $h_{1}$, ou par le nombre de points utilisés $(N=100$ à 200).

Le choix de $h_{0}$ est d'autant plus critique que l'équation (6) est un développement limité : les termes d'ordre supérieur en $\mathrm{e}^{-2 n \alpha h}$ ne peuvent être négligés que si :

$$
h_{0}>h_{0 \text { min }} \text {. }
$$

Il reste donc à déterminer $h_{0 \text { min }}$ dans le cas d'un interférogramme réel. Dans le souci de comparer des choses directement comparables, nous avons rassemblé dans le tableau II les quantités :

$$
\frac{\Delta \varepsilon^{\prime}}{\varepsilon_{\text {exacte }}^{\prime}}, \frac{\Delta \varepsilon^{\prime \prime}}{\varepsilon_{\text {exacte }}^{\prime \prime}}
$$

calculées pour des valeurs croissantes de $h_{0}$.

a) Dans le cas d'un interférogramme simulé, du type (6), correspondant à un pouvoir de résolution de 200 (soit sensiblement les conditions de l'expérience puisque nous mesurons des tensions de 0 à $1 \mathrm{mV}$ à $\pm 5 \mu \mathrm{V}$ près).

b) Dans le cas de l'interférogramme expérimental $\mathrm{du}$ chloroforme à $\lambda=4,358 \mathrm{~mm}, \theta=20^{\circ} \mathrm{C}$, pour lequel les valeurs les plus probables de $\varepsilon^{\prime}, \varepsilon^{\prime \prime}$ sont précisément les valeurs de $\varepsilon_{\text {exacte }}^{\prime}$, $\varepsilon_{\text {exacte }}^{\prime \prime}$ précédentes.

Le tableau II met en évidence pour $h_{0}<0,55 \mathrm{~mm}$ une dispersion des valeurs de $\varepsilon^{\prime \prime}$ et $\varepsilon^{\prime \prime}$ importante dans le cas $b$ ), inexistante au contraire dans le cas $a$ ). Cette dispersion caractérise l'influence non négligeable des termes en $\mathrm{e}^{-6 \alpha h}$ ou d'ordre supérieur, dans l'équation des interférogrammes pour $h_{0}<h_{0 \text { min }}$. Le seuil $h_{0 \max }$ défini précédemment semble se situer vers $h_{0}=2,05 \mathrm{~mm}$ pour $a$ ) et $b$ ). Nous prendrons donc pour valeurs extrêmes de $h_{0}$ :

$$
h_{0 \min }=0,55 \mathrm{~mm} ; \quad h_{0 \max }=2,05 \mathrm{~mm} \text {. }
$$

Il subsiste néanmoins une dispersion des résultats lorsque l'on choisit $h_{0}$ dans l'intervalle $\left(h_{0 \text { min }}, h_{0 \text { max }}\right)$. Elle traduit globalement :

- L'existence d'erreurs systématiques d'ordre expérimental : dérive de paramètres supposés constants (fréquence, puissance, température), non-linéarité des dispositifs de mesure, insuffisance de leur pouvoir de

\begin{tabular}{|c|c|c|c|c|c|c|}
\hline \multirow{3}{*}{$\begin{array}{l}\text { INGERTITUDES } \\
\text { RELATIVES }\end{array}$} & \\
\hline & 0,05 & 0,55 & 1,05 & 1,55 & 2,05 & 2,55 \\
\hline & - & - & - & - & - & - \\
\hline$\frac{\Delta \varepsilon^{\prime}}{\varepsilon_{\text {exacte }}^{\prime}}(\%)\left({ }^{a}\right)$ & $+0,07$ & $+0,10$ & $+0,02$ & $-0,10$ & $-0,37$ & $-0,42$ \\
\hline$\frac{\Delta \varepsilon^{\prime \prime}}{\varepsilon_{\text {exacte }}^{\prime \prime}}(\%)(a)$ & $-0,22$ & $-0,45$ & $-0,15$ & $-1,05$ & $-1,10$ & $-4,65$ \\
\hline$\frac{\Delta \varepsilon^{\prime}}{\varepsilon_{\text {exacte }}^{\prime}}(\%)(b)$ & $+1,10$ & $+0,50$ & $+0,01$ & $-0,40$ & $-1,60$ & - \\
\hline$\frac{\Delta \varepsilon^{\prime \prime}}{\varepsilon_{\text {exacte }}^{\prime \prime}}(\%)\left({ }^{b}\right)$ & $-2,80$ & $-0,10$ & $-0,01$ & $+0,40$ & $+3,30$ & - \\
\hline
\end{tabular}
résolution, etc.

TABLEAU II

(a) Résultats correspondant à l'interférogramme simulé.

$\left.{ }^{b}\right)$ Résultats correspondant à un interférogramme réel. 
TABLEAU III

\begin{tabular}{|c|c|c|c|c|c|c|}
\hline & $\varepsilon^{\prime}$ & $\varepsilon^{\prime \prime}$ & $\operatorname{tg} \delta$ & $\lambda(\mathrm{mm})$ & $\varepsilon_{0}$ & $\theta \circ \mathrm{C}$ \\
\hline & - & - & 一 & - & - & - \\
\hline Chloroforme & 2,605 & 0,854 & 0,33 & 4,358 & 4,81 & $20^{\circ} \mathrm{G}$ \\
\hline Bromure d'isoamyle & 2,478 & 0,723 & 0,29 & 4,352 & 5,92 & $25^{\circ} \mathrm{G}$ \\
\hline Alcool butylique tertiaire & 2,544 & 0,298 & 0,12 & 4,350 & 11,46 & $30^{\circ} \mathrm{C}$ \\
\hline Ether di-n-butylique .... & 2,200 & 0,305 & 0,14 & 4,342 & 3,07 & $22,5^{\circ} \mathrm{C}$ \\
\hline
\end{tabular}

- L'insuffisance du modèle mathématique (6) : on néglige en particulier l'influence des modes parasites et celle des termes du développement en $\mathrm{e}^{-6 \alpha h}$ ou d'ordre supérieur.

C'est pourquoi nous proposons de prendre pour incertitude absolue de nos résultats un majorant de la dispersion observée lorsque l'on fait varier $h_{0}$ dans le domaine $\left(h_{0 \min }, h_{0 \max }\right)$. Ceci nous conduit aux incertitudes relatives :

$$
\frac{\Delta \varepsilon^{\prime}}{\varepsilon^{\prime}}<1 \% ; \quad \frac{\Delta \varepsilon^{\prime \prime}}{\varepsilon^{\prime \prime}}<2 \%
$$

dans le cas du chloroforme $(b)$, alors que nous indiquions précédemment [1] des barres d'erreurs respectivement de $2 \%$ et $4 \%$.

Ainsi, l'utilisation de notre programme nous a permis de confirmer et de préciser les résultats que nous avions donnés pour différents corps [1] (tableau III).

Les incertitudes relatives obtenues sont toutes du même ordre de grandeur, soit :

$$
\frac{\Delta \varepsilon^{\prime}}{\varepsilon^{\prime}}<3 \% ; \quad \frac{\Delta \varepsilon^{\prime \prime}}{\varepsilon^{\prime \prime}}<2 \% .
$$

Nous avons pu vérifier également que la température n'était pas un facteur critique :

$$
\frac{1}{\varepsilon}\left[\frac{\partial \varepsilon}{\partial T}\right]<3 \% \text { par }{ }^{\circ} \mathrm{G} \text {. }
$$

En revanche, il est clair que les barres d'erreurs précédentes n'ont de sens que si l'on se donne un critère de pureté chimique, par exemple la constante diélectrique statique $\varepsilon_{0}$.

Conclusion. - Ainsi, une étude plus approfondie de l'interféromètre de Michelson nous a permis successivement :

- D'expliciter sans ambiguïté le rôle d'inévitables réflexions ou T.O.S. parasites.

- De montrer que la périodicité des interférogrammes à vide constituait un excellent critère de l'absence de modes supérieurs.

- De donner aux interférogrammes expérimentaux une forme analytique simple, indispensable à leur exploitation par une méthode numérique.

Dans ces conditions, nous avons développé une méthode d'optimisation de la permittivité complexe des liquides en ondes millimétriques. Elle se recom- mande par la rapidité de son traitement sur ordinateur, l'élimination de toute équation personnelle et surtout la possibilité d'une détermination semi-expérimentale des barres d'erreurs de ses résultats.

Ainsi, dans le cas des divers liquides étudiés à $\lambda \sim 4,35 \mathrm{~mm}$, nous avons pu réduire l'incertitude relative à $1 \%$ pour $\varepsilon^{\prime}$ et $2 \%$ pour $\varepsilon^{\prime \prime}$.

La réalisation de filtres de modes nous a permis par ailleurs d'étendre notre technique interférométrique au domaine des ondes centimétriques $(\nu>19 \mathrm{GHz})$. Des résultats très encourageants ont été obtenus dans ce domaine, également avec notre méthode de calcul; ils seront publiés prochainement.

Remerciements. - Nous remercions M. le Professeur Barriol de l'intérêt personnel qu'il a manifesté pour notre travail, réalisé avec l'aide matérielle du C.N.R.S., au laboratoire de Chimie Théorique de la Faculté des Sciences de Nancy.

\section{ANNEXE I}

Nous nous proposons de donner l'expression générale du coefficient de réflexion complexe $\rho_{2}$ mesuré dans le plan $\mathrm{P}_{2}$ (fig. 3), pour une épaisseur variable $h$ de liquide

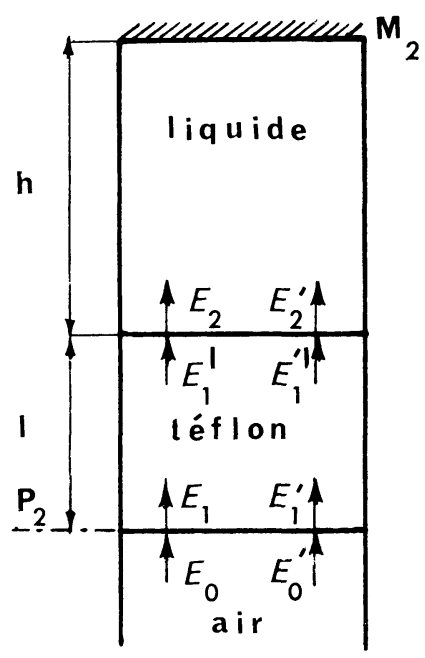

Fig. 3. - Schéma équivalent à la cellule de mesure : $h$, abscisse du piston de court-circuit $\mathrm{M}_{2} ; l$, épaisseur de la fenêtre de téflon; $\mathrm{P}_{2}$, Plan de référence du bras de mesure. 
dans la cellule. Le système étudié comprend successivement :

- Une discontinuité air-téflon (0-1).

- Un tronçon de ligne de longueur $l$ dans le téflon (1).

- Une discontinuité téflon-liquide (1-2).
- Un tronçon de ligne de hauteur $h$ dans le liquide (2).

- Un piston mobile jouant le rôle de miroir réfléchissant $\mathrm{M}_{2}$.

Il suffit d'écrire la continuité des champs électriques et magnétiques aux interfaces pour retrouver la relation matricielle :

$$
\left\|\begin{array}{c}
E_{0} \\
E_{0}^{\prime}
\end{array}\right\|=\frac{\left(\gamma_{0}+\gamma_{1}\right)\left(\gamma_{1}+\gamma_{2}\right)}{4 \gamma_{0} \gamma_{1}}\left\|\begin{array}{cc}
1 & r_{01} \\
r_{01} & 1
\end{array}\right\| \cdot\left\|\begin{array}{cc}
\mathrm{e}_{1} l & 0 \\
0 & \mathrm{e}^{\gamma_{1} l} \|
\end{array}\right\| \cdot\left\|\begin{array}{cc}
1 & r_{12} \\
r_{12} & 1
\end{array}\right\| \cdot\left\|\begin{array}{c}
E_{2} \\
E_{2}^{\prime}
\end{array}\right\|
$$

$\gamma_{i}$ étant la constante de propagation du milieu $i$ :

$$
r_{i j}=\frac{\gamma_{i}-\gamma_{j}}{\gamma_{i}+\gamma_{j}}
$$

Par ailleurs, nous avons la relation :

d'où :

$$
E_{2} \mathrm{e}^{-\gamma_{2} h}+E_{2}^{\prime} \mathrm{e}^{+\gamma_{2} h}=0
$$

$\rho_{2}(h)=\frac{E_{0}^{\prime}}{E_{0}}=\frac{r_{01}+r_{12} \mathrm{e}^{-2 \gamma_{1} l}-\left(r_{01} r_{12}+\mathrm{e}^{-2 \gamma_{1} l}\right) \cdot \mathrm{e}^{-2 \gamma_{2} h}}{1+r_{01} r_{12} \mathrm{e}^{-2 \gamma_{1} l}-\left(r_{12}+r_{01} \mathrm{e}^{-2 \gamma_{1} l}\right) \cdot \mathrm{e}^{-2 \gamma_{2} h}}$.
Il est possible de mettre $\rho_{2}(h)$ sous la forme d'une fonction homographique de $X(h)=\mathrm{e}^{-2 \gamma_{2} h}$ :

$$
\rho_{2}(h)=\frac{P-Q X}{1-R X} \quad \text { avec } \quad\left\{\begin{array}{l}
P=\frac{r_{01}+r_{12} \mathrm{e}^{-2 \gamma_{1} l}}{1-r_{01} r_{12} \mathrm{e}^{-2 \gamma_{1} l}} \\
Q=\frac{r_{01} r_{12}+\mathrm{e}^{-2 \gamma_{1} l}}{1-r_{01} r_{12} \mathrm{e}^{-2 \gamma_{1} l}} \\
R=\frac{r_{12}+r_{01} \mathrm{e}^{-2 \gamma_{1} l}}{1-r_{01} r_{12} \mathrm{e}^{-2 \gamma_{1} l}} .
\end{array}\right.
$$

\section{BIBLIOGRAPHIE}

[1] Goulon (J.), Roussy (G.) et Rivail (J. L.), Rev. Phys. Appl., 1968, 3, 231.

[2] Leroy (Y.), Barors (J. L.) et Constant (E.), C. $R$. Acad. Sci. Paris, 1966, 262 B, 1660-1663.

[3] Coumes (A.) et PIC (E.), L'onde électrique, 1965, 462, 1056-1061.

[4] BLED (J.), BREsson (A.), PAPOUlaR (R.) et WEGROOVE (J. G.), L'onde électrique, 1964, 442, 26-35.
[5] Schulten (V. G.), Frequenz, 1966, 20, 10-22.

[6] Gerschel (A.) et Brot (C.), Rev. Phys. Appl., 1966, 2, 113.

[7] Rosenbrock (H. H.), Comp. J., 1960, 3, 175-84.

[8] LINNIK (Y. V.), Méthodes des moindres carrés, Dunod, Paris, 1963.

[9] Goudet (G.) et Chavance (P.), Ondes centimétriques, Éditions Chiron, Paris, 1955. 